\title{
Minireview
}

\section{AMNIO7 (nilotinib): a novel and selective inhibitor of BCR-ABL}

\author{
E Weisberg', P Manley², J Mestan², S Cowan-Jacob², A Ray' and JD Griffin ${ }^{*, 1}$ \\ 'Department of Adult Oncology, Dana Farber Cancer Institute, 44 Binney Street, Boston, MA 021 I 5, USA; ${ }^{2}$ Novartis Institutes of Biomedical Research,
} Basel, Switzerland

\begin{abstract}
Chronic myelogenous leukaemia (CML) and Philadelphia chromosome positive $(\mathrm{Ph}+)$ acute lymphoblastic leukaemia $(\mathrm{ALL})$ are caused by the $B C R-A B L$ oncogene. Imatinib inhibits the tyrosine kinase activity of the $B C R-A B L$ protein and is an effective, frontline therapy for chronic-phase CML. However, accelerated or blast-crisis phase CML patients and Ph + ALL patients often relapse due to drug resistance resulting from the emergence of imatinib-resistant point mutations within the $B C R-A B L$ tyrosine kinase domain. This has stimulated the development of new kinase inhibitors that are able to over-ride resistance to imatinib. The novel, selective $B C R$ $A B L$ inhibitor, $A M N 107$, was designed to fit into the ATP-binding site of the BCR-ABL protein with higher affinity than imatinib. In addition to being more potent than imatinib $(I C 50<30 \mathrm{nM})$ against wild-type $B C R-A B L$, AMNIO7 is also significantly active against 32/33 imatinib-resistant BCR-ABL mutants. In preclinical studies, AMNIO7 demonstrated activity in vitro and in vivo against wild-type and imatinib-resistant BCR-ABL-expressing cells. In phase 1/II clinical trials, AMNI07 has produced haematological and cytogenetic responses in CML patients, who either did not initially respond to imatinib or developed imatinib resistance. Dasatinib (BMS-354825), which inhibits Abl and Src family kinases, is another promising new clinical candidate for CML that has shown good efficacy in CML patients. In this review, the early characterisation and development of AMNI07 is discussed, as is the current status of AMN 07 in clinical trials for imatinib-resistant $\mathrm{CML}$ and $\mathrm{Ph}+\mathrm{ALL}$. Future trends investigating prediction of mechanisms of resistance to AMNI07, and how and where AMNIO7 is expected to fit into the overall picture for treatment of early-phase CML and imatinibrefractory and late-stage disease are discussed.

British Journal of Cancer (2006) 94, 1765-1769. doi: I0.1038/sj.bjc.6603170 www.bjcancer.com
\end{abstract}

Published online 23 May 2006

(C) 2006 Cancer Research UK

Keywords: BCR-ABL; AMN I07; nilotinib; dasatinib; imatinib-resistance

Chronic myelogenous leukaemia (CML) constitutes 15\% of adult leukaemias, with approximately 4600 newly diagnosed cases per annum in the United States. The initial, chronic phase of the disease has a median duration of $4-6$ years and is characterised by overproduction of immature myeloid cells and mature granulocytes in the spleen, bone marrow, and peripheral blood. Without therapeutic intervention, after a mean latency period of 4-6 years, the disease progresses via an accelerated phase, marked by the presence of primitive blast cells in the bone marrow and peripheral blood, and finally advances to the 'blast-crisis' phase, characterised by over $30 \%$ undifferentiated blasts in the bone marrow and peripheral blood, and for which median survival is 18 weeks (Kantarjian and Talpaz, 1988).

The $B C R-A B L$ oncogene, which results from a reciprocal $t(9 ; 22)$ chromosomal translocation, encodes a chimeric $B C R-A B L$ protein having constitutively activated Abl tyrosine kinase activity, and is the underlying cause of CML (Bartram et al, 1983; Groffen et al, 1984 ; Lugo et al, 1990). The $210 \mathrm{kDa} B C R-A B L$ protein is expressed in CML patients, whereas a $190 \mathrm{kDa} B C R-A B L$ protein, resulting from an alternative breakpoint in the $B C R$ gene, is expressed in $\mathrm{Ph}+$ acute lymphoblastic leukaemia (ALL) patients (Chan et al, 1987).

*Correspondence: Dr JD Griffin; E-mail: James_Griffin@dfci.harvard.edu Several of the authors are employed by a company (Novartis) whose product is described in the present work.

Received I5 February 2006; revised 20 April 2006; accepted 21 April 2006; published online 23 May 2006
The discovery that CML is due to the activity of $B C R-A B L$ prompted the design and development Novartis Pharma AG, WKL-136.7.86, Klybeckstrasse 141, CH-4057 Basel, Switzerland of imatinib (Glivec ${ }^{\mathbb{R}}$, Gleevec ${ }^{\mathrm{TM}}$, STI571; Novartis Pharma AG), a small molecule kinase inhibitor that targets the PDGFR, c-Kit and Abl kinases (Druker et al, 1996; Buchdunger et al, 2000). Imatinib provides an effective and durable therapy for $\mathrm{CML}$, inducing complete haematologic remissions (normal leucocyte count in peripheral blood) in the majority (98\%) of newly diagnosed patients in the chronic phase of the disease, and complete cytogenetic responses (no detectable $\mathrm{Ph}+$ cells from $\geqslant 20$ bone marrow cells in metaphase) in a high percentage $(86 \%)$ of patients (Simonsson, 2005). Primary resistance to imatinib only occurs occasionally in chronic-phase CML patients, and recent analysis of the IRIS study shows a low and decreasing annual rate of progression (resulting in death) after 1, 2, 3 and 4 years of therapy of $3.4,7.5,4.8$ and $1.5 \%$, respectively, possibly as a result of patients with the worse prognosis progressing relatively early. In $39 \%$ of newly diagnosed chronic-phase CML patients, therapy with a standard dose of imatinib for 12 months leads to a major molecular response comprising of 1000 -fold reduction in $B C R-A B L$ transcript levels, which is associated with a reduced risk of disease progression (Hughes et al, 2003). However, advanced (accelerated or blast crisis) phase CML and $\mathrm{Ph}+\mathrm{ALL}$ patients show significantly decreased response rates to treatment with imatinib monotherapy, with relapse common within a year (Ottmann et al, 2002; Sawyers et al, 2002); acquired resistance is less commonly observed in the case of newly diagnosed $\mathrm{Ph}+$ ALL patients receiving combination therapy with chemotherapy. 
Resistance frequently results from the emergence of point mutations within the kinase domain of the $B C R-A B L$ protein that reduce the binding affinity of imatinib, although it is occasionally associated with amplification of the $B C R-A B L$ gene (Gorre et al, 2001). Most mutations that confer resistance to imatinib are distributed throughout the Abl kinase domain. However, the most resistant ones, such as many of those found in the P-loop, often occur at or near residues that are in direct contact with the drug. The degree of resistance ranges from a few fold for some of the A-loop mutants, up to complete resistance for the T315I mutation, which precludes imatinib from binding. Overall, the steady rate of developing resistance to imatinib has suggested that new kinase inhibitors could be of clinical value, particularly if they could override imatinib resistance and bind with higher affinity to $B C R-A B L$.

\section{AMN107 (NILOTINIB)}

Rational design of novel inhibitors exhibiting effectiveness against imatinib-resistant mutants of $B C R-A B L$ was carried out by researchers at Novartis Pharmaceuticals, based upon the crystal structure of the imatinib-Abl complex (Schindler et al, 2000; Nagar et al, 2002; Manley et al, 2004). It was hypothesised that the potency and selectivity of imatinib (Figure 1F) could be improved by maintaining binding to the inactive conformation of the $\mathrm{Abl}$ kinase domain, but incorporating alternative binding groups to the
$N$-methylpiperazine moiety, while preserving an amide pharmacophore to retain H-bond interactions to Glu286 and Asp381. This led to the development of AMN107 (nilotinib; Figure 1E), a highaffinity aminopyrimidine-based ATP-competitive inhibitor that decreases proliferation and viability of wild-type $B C R-A B L$ - and imatinib-resistant $B C R-A B L$ mutant-expressing cells in vitro by selectively inhibiting $B C R-A B L$ autophosphorylation (Table 1). AMN107 exhibits superior potency to imatinib as an inhibitor of wild-type $B C R-A B L$ in a wide range of CML-derived and transfected cell lines (Golemovic et al, 2005; Weisberg et al, 2005). This in vitro profile translates into in vivo efficacy, where AMN107 has been shown to prolong the survival of mice injected with $B C R-A B L$-transformed haematopoietic cell lines or primary marrow cells, and to prolong survival in imatinib-resistant CML mouse models (Weisberg et al, 2005).

As well as being designed to bind more tightly to the $B C R-A B L$ protein in an attempt to enhance efficacy, AMN107 was intended to over-ride resistance caused by mutations. Crystallographic studies of AMN107 indeed suggest that subtle differences in the mode of binding to $\mathrm{Abl}$ and a better topological fit to the $\mathrm{Abl}$ protein account for the greater potency of the drug (Weisberg et al, 2005). Like imatinib, AMN107 binds to the inactive conformation of the Abl tyrosine kinase, with P-loop folding over the ATPbinding site, and the activation-loop blocking the substrate binding site, to disrupt the ATP-phosphate-binding site and inhibit the catalytic activity of the enzyme (Figure 1C) (Manley et al, 2005). AMN107 makes four hydrogen-bond interactions with
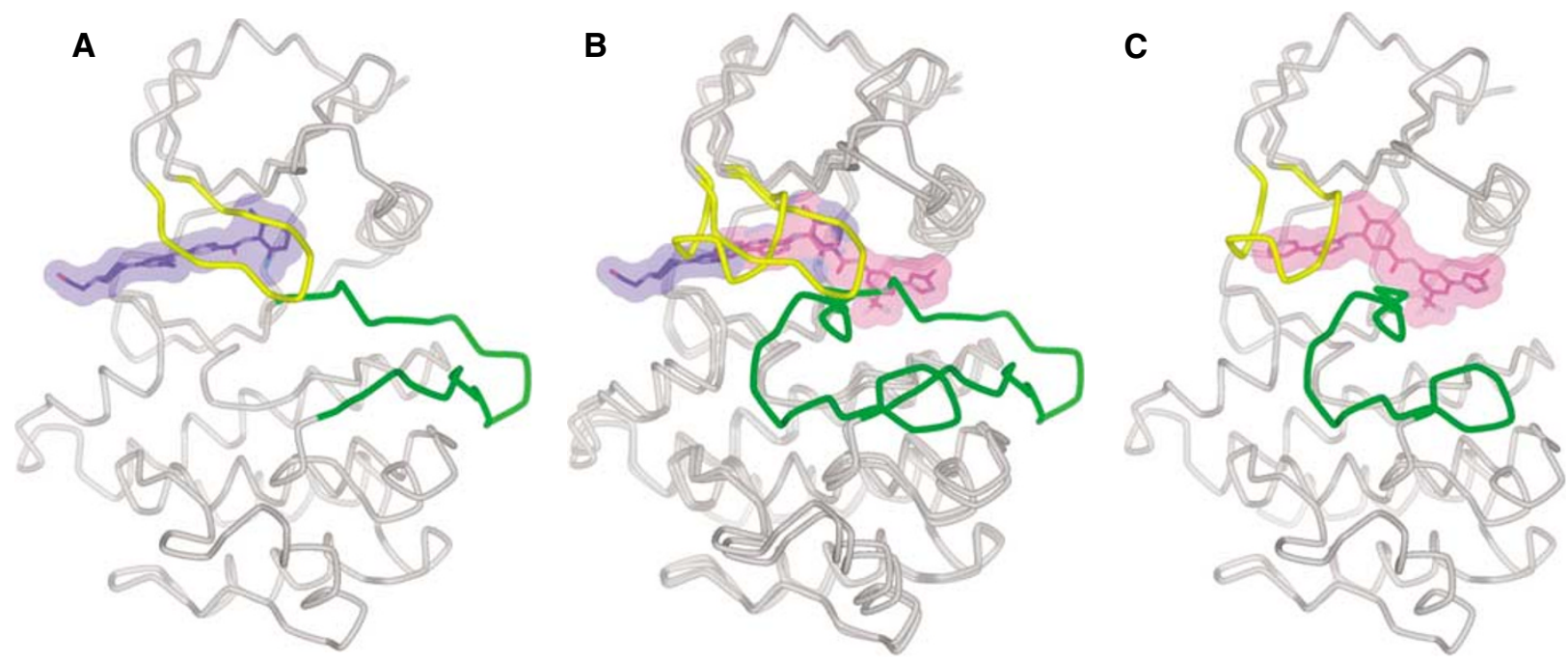

D

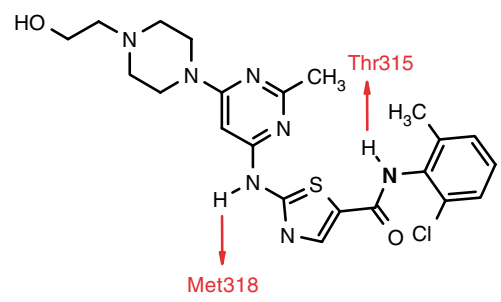

$\mathbf{E}$

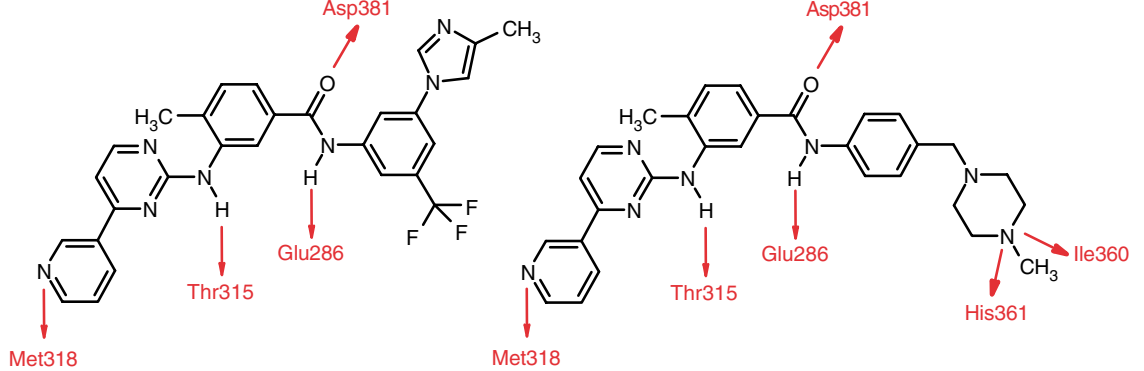

Figure I Structures of Abl kinase $(\mathbf{A})$ in the active (Fendrich et al, 2006) and $(\mathbf{C})$ inactive states, with dasatinib (blue) docked and nilotinib (magenta) as bound in the crystal structure (Weisberg et al, 2005), respectively. The differing conformations of the glycine-rich or P-loop (yellow) and the activation loop (green) are induced or stabilised by the different binding modes of the two inhibitors. (B) shows a superposition of the two distinct conformations, emphasising how dasatinib and nilotinib occupy different parts of the cleft between the $\mathrm{N}$ - (upper) and C-terminal (lower) lobes of the kinase. The corresponding aspects of the molecular structures of $(\mathbf{D})$ dasatinib and $(\mathbf{E})$ nilotinib are depicted, with their respective $\mathrm{H}$-bond interactions with the Abl kinase domain indicated in red, in comparison to imatinib (F). 
Table I Comparison of imatinib and AMNI 07 for effects on autophosphorylation and proliferation in Ba/F3 cells transfected to express native BCR-ABLor imatinib-resistant mutant forms of the enzyme

\begin{tabular}{|c|c|c|c|c|}
\hline \multirow[b]{2}{*}{$B C R-A B L$ form (construct) } & \multicolumn{2}{|c|}{ Imatinib } & \multicolumn{2}{|c|}{ AMNIO7 } \\
\hline & Autophosphorylation & Proliferation & Autophosphorylation & Proliferation \\
\hline Wild-type p210 & $22 \mid \pm 31(14)$ & $678 \pm 39(23)$ & $20 \pm 2(7)$ & $25 \pm 1(68)$ \\
\hline M237I (p|85) & $399(2)$ & $1545(2)$ & $4 I \pm 8.3(3)$ & $43 \pm 8.7$ (3) \\
\hline M244V (p|85) & 937 (2) & 2036 (2) & $101 \pm 16(3)$ & $67 \pm 7(4)$ \\
\hline L248V (p|85) & $101 \mid(2)$ & $208 \mid(2)$ & $83 \pm 7(3)$ & $102 \pm 13(4)$ \\
\hline G250V (p|85) & $489(2)$ & $624(2)$ & $66 \pm 12(3)$ & $19 \pm 1.4(3)$ \\
\hline Q252H (p|85) & $1080 \pm 119(2)$ & $85 I \pm 436(2)$ & $117 \pm 25(3)$ & $67 \pm 22(4)$ \\
\hline Y253H (p/85) & $>10000(2)$ & $>7000(2)$ & $260 \pm 34(6)$ & $700 \pm 116(5)$ \\
\hline E255D (p|85) & $754(2)$ & $1082(2)$ & $5 I \pm 4.8$ & $27 \pm 3.1$ \\
\hline E255K (p|85) & $4856 \pm 482(4)$ & $5567(2)$ & $392 \pm 82(6)$ & $308 \pm 42(5)$ \\
\hline E255K (p210) & $2455 \pm 433$ (4) & $7161 \pm 970$ & $153 \pm 9(4)$ & $548 \pm 72(6)$ \\
\hline E255R (p / 85) & $1877(2)$ & $1 \overline{567}(2)$ & $240 \pm 6.5(3)$ & $58 \pm 4.2(3)$ \\
\hline E292K (p2।0) & $275 \pm 81$ (3) & 1552 (2) & $31 \pm 6(3)$ & $81 \pm 8(4)$ \\
\hline F3IIV (p|85) & $1480(2)$ & $3535(2)$ & $84 \pm 2(3)$ & $155 \pm 31(4)$ \\
\hline T3।5I (p2|0) & $>10000(22)$ & $>7000(17)$ & $>10000(48)$ & $>10000(51)$ \\
\hline F3|7C (p|85) & $1090(2)$ & $694(2)$ & $69 \pm 13(3)$ & $20 \pm 3.1$ \\
\hline F3|7L (p2|0) & $797 \pm 92($ (II) & $1528 \pm 227(15)$ & $38 \pm 4(13)$ & $91 \pm 6.5(17)$ \\
\hline F3|7V (p|85) & $544 \pm 47(3)$ & $549 \pm 173(4)$ & $95 \pm 28(3)$ & $28 \pm 4(4)$ \\
\hline D325N" (p|85) & $\overline{5} 84(2)$ & $\overline{887}(2)$ & $70 \pm 9.0(3)$ & $26 \pm 2.7(3)$ \\
\hline S348L (p|85) & $553(2)$ & $1370(2)$ & $55 \pm 1.3(3)$ & $26 \pm 4.8(3)$ \\
\hline M35IT (p2।0) & $593 \pm 57(\mathrm{II})$ & $1682 \pm 233(18)$ & $29 \pm 3(13)$ & $38 \pm 4(18)$ \\
\hline E355A (p/85) & $676(2)$ & $1 \overline{434}(2)$ & $90 \pm 17(3)$ & $35 \pm 6.7(3)$ \\
\hline E355G (p|85) & $601(2)$ & I 49 (2) & $67 \pm 15(3)$ & $47 \pm 8(4)$ \\
\hline F359C (p|85) & $1130(2)$ & $2377(2)$ & $217 \pm 17(3)$ & $258 \pm 61(3)$ \\
\hline F359V (p|85) & $1528(2)$ & 595 (2) & $313 \pm 79$ (3) & $\mid 61 \pm 61$ (4) \\
\hline
\end{tabular}

The influence of compounds on kinase autophosphorylation or cell viability was calculated as percentage inhibition as described (Weisberg et al, 2005). Dose-response curves were used to calculate $I C_{50}$ values, expressed as mean \pm s.e.m. (nM) (number of replicates). The influence of compounds on BCR-ABL autophosphorylation or cell viability was determined with capture ELISAs or the ATPlite ${ }^{\mathrm{TM}}$ assay kit (Perkin-Elmer), respectively. Dose-response curves (per cent inhibition) were used to calculate $I_{50}$ values, expressed as mean \pm s.e.m., $n=$ number of experiments.

the Abl kinase domain (Figure 1E), involving the pyridyl-N and the backbone-NH of Met318, the anilino-NH and the side-chain hydroxyl of Thr315, the amido-NH and side-chain carboxylate of Glu286, as well as the amido- $\mathrm{C}=\mathrm{O}$ and backbone- $\mathrm{NH}$ of Asp381, to induce the inactive conformation of $B C R-A B L$ (Figure 1C) (Manley et al, 2005). However, the many lipophilic interactions are also important for affinity, as is the interaction between the backbone- $\mathrm{C}=\mathrm{O}$ of Asp381 and a fluorine atom in the trifluoromethyl group of AMN107 (Manley et al, 2005).

AMN107 is $\geqslant 20$-fold more potent than imatinib in the killing of wild-type $B C R-A B L$-expressing cells (Table 1) (Manley et al, 2005; O'Hare et al, 2005; Weisberg et al, 2005). Studies involving the imatinib-sensitive cell lines KBM5 and KBM7 show AMN107 to be 43- and 60 times more potent than imatinib, respectively (Golemovic et al, 2005). AMN107 maintains activity against $32 / 33$ imatinib-resistant $B C R-A B L$ mutants, but has no significant activity against the T315I mutant (Table 1) (Manley et al, 2005; O'Hare et al, 2005; Weisberg et al, 2005). As with imatinib, the lack of activity against the T315I mutant is the result of AMN107 binding closely to the T315 residue, such that loss of the hydroxyl side chain and additional methyl group of the isoleucine inhibits binding (Figure 1E and $\mathrm{F}$ ).
In a dose-escalating Phase I study, imatinib-resistant CML patients in the chronic phase (17 patients), accelerated phase (46 patients) and blast crisis (33 patients), together with $13 \mathrm{Ph}+\mathrm{ALL}$ patients, were treated with AMN107 (50-1200 $\mathrm{mg} \mathrm{day}^{-1}$ ) for up to 385 days (Kantarjian et al, 2006). The maximum tolerated dose was determined to be $600 \mathrm{mg}$ b.i.d., with frequently noted side effects being myelosuppression, mild-moderate skin rash and transient indirect hyperbilirubinaemia. In this study, AMN107 was not associated with the oedema frequently associated with imatinib. Among patients with chronic, accelerated and blast-phase CML, haematological/cytogenetic responses were achieved in 92/53, $72 / 48$ and $39 / 27 \%$, respectively. The best responses were seen at doses $\geqslant 400 \mathrm{mg}$ q.d. and with $400 \mathrm{mg}$ b.i.d. Two of the imatinibresistant $\mathrm{Ph}+\mathrm{ALL}$ patients also responded. Pharmacokinetic analysis of patients receiving $400 \mathrm{mg}$ b.i.d., which was the dose selected for Phase II trials, showed mean peak-trough plasma levels of 3.6 and $1.7 \mu \mathrm{M}$, respectively, with an apparent half-life of $15 \mathrm{~h}$. Based upon the in vitro data, this level of drug exposure would be expected to result in clinical activity against most of the mutants characterised in Table 1, with the exception of T315I, and is therefore consistent with the responses observed in patients harbouring imatinib-resistant point mutations. 
The early-phase clinical trials, therefore, support the possibility that AMN107 will have substantial clinical utility in rescuing patients who develop imatinib resistance due to point mutations, and could potentially be used as a single agent in patients at risk for progression. Additionally, there is growing interest in testing the hypothesis that administration of multiple Abl kinase inhibitors in early-phase patients, such as AMN107, dasatinib (Shah et al, 2005) and imatinib, could be used to delay or prevent the emergence of drug-resistant clones. In support of these ideas, additive/synergistic toxicity against both imatinib-sensitive and imatinib-resistant $B C R-A B L$-expressing cells has been reported following coadministration of AMN107 and imatinib, in vitro and in vivo (Griffin and Weisberg, 2005; Weisberg et al, 2005). Such effects might result from pharmacodynamic effects, and preliminary data suggest that synergy between imatinib and AMN107 may occur at the level of the CML stem cell due to the ability of both imatinib and AMN107 to inhibit or act as substrates of the multidrug efflux transporter ABCG2, which confers resistance toward several anticancer drugs (Jorgensen et al, 2005). A recent report also suggests that imatinib and AMN107 are taken up in cells by different mechanisms, with the influx, intracellular concentrations of imatinib, and consequently patient sensitivity to imatinib depending upon the organic cation transporter Oct-1, whereas AMN107 transport appears to be independent of Oct-1 (White et al, 2006). However, since the T315I mutation of BCR$A B L$ is highly resistant to imatinib, AMN107 and dasatinib, this approach needs to be extended to include inhibitors of T315I BCR$A B L$ to prevent this mutation from becoming more prevalent. Alternatively, it is also important to explore the potential for synergy between AMN107 and other classes of inhibitors that work through mechanisms not involving inhibition of Abl tyrosine kinase activity.

To aid the selection of patients most likely to benefit and show clinical responses to single agents, as well as to assess which drug combinations might be most appropriate, it is important to be able to predict resistance mechanisms and establish the resistance profiles of the available $B C R-A B L$ inhibitors. Although overexpression of $B C R-A B L$ is a possible resistance mechanism for AMN107 (Mahon et al, 2004), resistance is more likely to arise through the emergence of clones expressing AMN107-resistant mutant forms of BCR-ABL. A cell-based screening assay designed to predict such mutations has recently been applied to AMN107 (Von Bubnoff et al, 2006). Using this system, a reduced pattern of mutations was observed for AMN107, having some overlap with that seen for imatinib: Q252H, Y253H, E255K(V), F311I, T315I, S349L and F359I(V), all of which, with the exception of the T315I mutant, were suppressed at clinically achievable concentrations of AMN107. In an alternative cell-line-based mutagenesis study, the emergence of $B C R-A B L$ mutations resistant to imatinib, AMN107 and dasatinib were compared: 18 mutations were recovered with imatinib, nine mutations (G250E, Y253H, E255K(V), E292V, T351I, F359C, L384M and L387F) were recovered with AMN107, and six mutations (E255K, L284V, V299L, T315I and F317I(V)) were recovered with dasatinib (Deininger et al, 2005). In a similar mutagenesis study with dasatinib (Shah et al, 2005), 10 resistance mutants of $B C R-A B L$ involving six residues were isolated: $L 248 \mathrm{R}$, Q252H, E255K, V299L, F317L/V/I/S and T315I/A. BCR-ABL point mutations conferring resistance to AMN107 have also been identified in a random mutagenesis study (Ray et al, 2005). In this study, 11 novel mutations were detected (K247N, L248V, L273F, E282K, K285N, V289L, E292K, N297T, H375P, T406I and W430L), in addition to five (Q252H, Y253C(H), E255K and T315I), which have been previously observed in CML patients treated with imatinib. Although these studies do not consistently identify the same drug-resistant $B C R-A B L$ point mutations for individual drugs, it is clear that all three compounds display different mutagenicity profiles.
Since the pattern of arising $B C R-A B L$ mutants should be associated with the binding mode of that particular compound to the Abl protein, conceptually, the greatest benefit from a combination of two such agents should be achieved using compounds having the greatest difference between their binding modes. Thus, whereas both imatinib and AMN107 bind to an inactive conformation of Abl (Figure 1C), dasatinib has been shown to bind to the active conformation (Figure 1A), and this can be invoked to explain the differences observed in the mutagenesis studies with these compounds. Therefore, a combination between dasatinib and AMN107 (or imatinib) might be expected to impart the greatest benefit (cf. Figure 1B), since dasatinib might inhibit many AMN107/imatinib-resistant mutants and conversely AMN107/imatinib might inhibit many dasatinibresistant mutants.

Other studies have uncovered additional targets of AMN107 that help to elucidate its mechanism of action and/or suggest additional disease targets. Both AMN107 and imatinib have been observed to promote the expression of Bcl-2-interacting mediator, a tumour suppressor reported to be underexpressed in primary CML cells in comparison to normal cells (Aichberger et al, 2005). The ability of AMN107 to inhibit TEL-platelet-derived growth factor receptor-beta (TEL-PDGFRbeta), which causes chronic myelomonocytic leukaemia, and FIP1-like-1-PDGFRalpha, which causes hypereosinophilic syndrome, suggests potential use of AMN107 for myeloproliferative diseases characterised by these kinase fusions (Stover et al, 2005; Weisberg et al, 2005). AMN107 also inhibits the c-Kit receptor kinase, including the D816V-mutated variant of KIT, at pharmacologically achievable concentrations, supporting potential utility in the treatment of mastocytosis, and gastrointestinal stromal tumours (Weisberg et al, 2005; von Bubnoff et al, 2005; Gleixner et al, 2006).

\section{CONCLUSION}

Preclinical and early-phase clinical findings indicate that AMN107 may be useful in the treatment of imatinib-refractory CML. This is due to its strong binding affinity to Abl, its activity against imatinib-resistant $B C R-A B L$ point mutants, and its efficacy and tolerability in clinical studies. Greater than $70 \%$ of CML patients with advanced disease and over $90 \%$ of early, chronic-phase patients have responded to AMN107, and response rates continue to increase with overall good tolerability. In order to evaluate AMN107 in newly diagnosed CML, a study has recently been initiated (MD Anderson Cancer Center, Houston).

The failure of some patients to respond to AMN107, especially those with more advanced disease, might arise due to development of new mutations that impede the interaction between AMN107 and $B C R-A B L$. Thus, the identification and characterisation of $B C R-A B L$ point mutants conferring resistance to AMN107 will assist in the prediction of patient responses to AMN107, identifying combination partners, as well as in the design and development of novel inhibitors of $B C R-A B L$ that can over-ride resistance to such mutants. The preclinical and clinical evaluation of combinations of AMN107 with other approved or investigational inhibitors of $\mathrm{Abl}$ and additional signaling pathways will be helpful in the development of therapeutic strategies designed to over-ride drug resistance.

Both the safety and effectiveness of AMN107 are currently being evaluated in clinical trials involving CML patients that are intolerant of, or refractory to, imatinib. Thus far, AMN107 is showing promise as a potential therapeutic for CML at all levels of the disease. The frequency of use of AMN107 as a treatment for CML and Ph + ALL will depend on its safety/efficacy profiles in clinical trials. 


\section{REFERENCES}

Aichberger KJ, Mayerhofer M, Krauth MT, Vales A, Kondo R, Derdak S, Pickl WF, Selzer E, Deininger M, Druker BJ, Sillaber C, Esterbauer H, Valent P (2005) Low-level expression of proapoptotic Bcl-2-interacting mediator in leukemic cells in patients with chronic myeloid leukaemia: role of $B C R$ $A B L$, characterization of underlying signaling pathways, and reexpression by novel pharmacologic compounds. Cancer Res 65: $9436-9444$

Bartram CR, de Klein A, Hagemeijer A, van Agthoven T, Geurts van Kessel A, Bootsma D, Grosveld G, Ferguson-Smith MA, Davies T, Stone M, Heisterkamp N, Stephenson JR, Groffen J (1983) Translocation of c-abl oncogene correlates with the presence of a Philadelphia chromosome in chronic myelocytic leukaemia. Nature 306: 277-280

Buchdunger E, Cioffi CL, Law N, Stover D, Ohno-Jones S, Druker BJ, Lydon NB (2000) Abl protein-tyrosine kinase inhibitor STI571 inhibits in vitro signal transduction mediated by c-Kit and platelet-derived growth factor receptors. J Pharmacol Exp Ther 295: 139-145

Chan LC, Karhi KK, Rayter SI, Heisterkamp N, Eridani S, Powle R, Lawler SD, Groffen J, Foulkes JG, Greaves MF, Wiedemann LM (1987) A novel abl protein expressed in Philadelphia chromosome positive acute lymphoblastic leukaemia. Nature 325: 635-637

Deininger MWN, Bradeen H, Jia T, O'Hare T, Willis SG, Lee F, Druker BJ (2005) Comparison of imatinib, AMN107, and dasatinib in an accelerated cell-based mutagenesis screen. Blood 106: 204a

Druker BJ, Tamura S, Buchdunger E, Ohno S, Segal GM, Fanning S, Zimmermann J, Lydon NB (1996) Effects of a selective inhibitor of the $\mathrm{Abl}$ tyrosine kinase on the growth of Bcr-Abl positive cells. Nat Med 2: $561-566$

Fendrich G, Jacob SW, Klon AE, Manley PW (2006) Three-dimensional structure of human c-Abl kinase in complex with anti-tumor ligand, and use in drug discovery. US Patent Appl. Pub. 2006030017, April 9

Gleixner KV, Mayerhofer M, Aichberger KJ, Derdak S, Sonneck K, Bohm A, Gruze A, Samorapoompichit P, Manley PW, Fabbro D, Pickl WF, Sillaber C, Valent P (2006) PKC412 inhibits in vitro growth of neoplastic human mast cells expressing the D816V-mutated variant of KIT: comparison with AMN107, imatinib, and cladribine (2CdA), and evaluation of cooperative drug effects. Blood 107: $752-759$

Golemovic M, Verstovsek S, Giles F, Cortes J, Manshouri T, Manley PW, Mestan J, Dugan M, Alland L, Griffin JD, Arlinghaus RB, Sun T, Kantarjian H, Beran M (2005) AMN107, a novel aminopyrimidine inhibitor of Bcr-Abl, has in vitro activity against imatinib-resistant chronic myeloid leukaemia. Clin Cancer Res 11: 4941 - 4947

Gorre ME, Mohammed M, Ellwood K, Hsu N, Paquette R, Rao PN, Sawyers CL (2001) Clinical resistance to STI-571 cancer therapy caused by BCR$A B L$ gene mutation or amplification. Science 293: $876-880$

Griffin JD, Weisberg E (2005) Simultaneous administration of AMN107 and imatinib in the treatment of imatinib-sensitive and imatinib-resistant chronic myeloid leukaemia. Blood 106: 205a

Groffen J, Stephenson JR, Heisterkamp N, de Klein A, Bartram CR, Grosveld G (1984) Philadelphia chromosomal breakpoints are clustered within a limited region, bcr, on chromosome 22. Cell 36: 93-99

Hughes TP, Kaeda J, Branford S, Rudzki Z, Hochhaus A, Hensley ML, Gathmann I, Bolton AE, van Hoomissen IC, Goldman JM, Radich JP (2003) Frequency of major molecular responses to imatinib or interferon alfa plus cytarabine in newly diagnosed chronic myeloid leukemia. New Engl J Med 349: $1423-1432$

Jorgensen H, Allan E, Jordanides N, Hamilton A, Mountford J, Holyoake T (2005) AMN107 appears equipotent and may synergise with imatinib at the CML stem cell level through interaction with ABCG2. Blood 106: 314a

Kantarjian H, Giles F, Wunderle L, Bhalla K, O’Brien S, Wassmann B, Tanaka C, Manley P, Rae P, Mietlowski W, Bochinski K, Hochhaus A, Griffin JD, Holzer D, Albitar M, Dugan M, Cortes J, Alland L, Ottman OG (2006) AMN107, a novel, highly active, selective Bcr-Abl tyrosine kinase inhibitor in patients with Philadelphia Chromosome $(\mathrm{Ph})$ positive chronic myelogenous leukaemia (CML) or acute lymphocytic leukaemia (ALL) who are resistant to imatinib mesylate therapy. New Engl J Med (in press)

Kantarjian HM, Talpaz M (1988) Definition of the accelerated phase of chronic myelogenous leukaemia. J Clin Oncol 6: 180-182

Lugo TG, Pendergast AM, Muller AJ, Witte ON (1990) Tyrosine kinase activity and transformation potency of bcr-abl oncogene products. Science 247: 1079 - 1082

Mahon FX, Lagarde V, Manley PW, Pasquet J-M, Turcq B, Reiffers J (2004) Generation of resistance cell lines to AMN107, a new inhibitor of Bcr-
$\mathrm{Abl}$, and its effects on cell lines sensitive and resistant to imatinib. Blood 104: $251 \mathrm{a}$

Manley PW, Breitenstein W, Bruggen J, Cowan-Jacob SW, Furet P, Mestan J, Meyer T (2004) Urea-derivatives of STI571 as inhibitors of Bcr-Abl and PDGFR kinases. Bioorg Med Chem Lett 14: 5793-5797

Manley PW, Cowan-Jacob SW, Fendrich G, Metan J (2005) Molecular interactions between the highly selective pan-Bcr-Abl inhibitor, AMN107, and the tyrosine kinase domain of Abl. Blood 106: 940a

Nagar B, Bommann WG, Pellicena P, Schindler T, Veach DR, Miller WT, Clarkson B, Kuriyan J (2002) Crystal structures of the kinase domain of c-Abl in complex with the small molecule inhibitors PD173955 and imatinib (STI-571). Cancer Res 62: 4236-4243

O'Hare T, Walters DK, Stoffregen EP, Jia T, Manley PW, Mestan J, CowanJacob SW, Lee FY, Heinrich MC, Deininger MW, Druker BJ (2005) In vitro activity of Bcr-Abl inhibitors AMN107 and BMS-354825 against clinically relevant imatinib-resistant Abl kinase domain mutants. Cancer Res 65: $4500-4505$

Ottmann OG, Druker BJ, Sawyers CL, Goldman JM, Reiffers J, Silver RT, Tura S, Fischer T, Deininger MW, Schiffer CA, Baccarani M, Gratwohl A, Hochhaus A, Hoelzer D, Fernandes-Reese S, Gathmann I, Capdeville R, O'Brien SG (2002) A phase 2 study of imatinib in patients with relapsed or refractory Philadelphia chromosome-positive acute lymphoid leukemias. Blood 100: 1965-1971

Ray A, Cowan-Jacob S, Manley PW, Mestan J, Griffin JD (2005) Identification of $B C R-A B L$ point mutations conferring resistance to the Abl kinase inhibitor AMN107 by a random mutagenesis study. Blood 106: $148 \mathrm{a}$

Sawyers CL, Hochhaus A, Feldman E, Goldman JM, Miller CB, Ottmann OG, Schiffer CA, Talpaz M, Guilhot F, Deininger MW, Fischer T, O'Brien SG, Stone RM, Gambacorti-Passerini CB, Russell NH, Reiffers JJ, Shea TC, Chapuis B, Coutre S, Tura S, Morra E, Larson RA, Saven A, Peschel C, Gratwohl A, Mandelli F, Ben-Am M, Gathmann I, Capdeville R, Paquette RL, Druker BJ (2002) Imatinib induces hematologic and cytogenetic responses in patients with chronic myelogenous leukaemia in myeloid blast crisis: results of a phase II study. Blood 99: 3530-3539

Schindler T, Bommann W, Pellicena P, Miller WT, Clarkson B, Kuriyan J (2000) Structural mechanism for STI-571 inhibition of abelson tyrosine kinase. Science 289: $1938-1942$

Shah NP, Nicoll JM, Branford S, Hughes TP, Paquette RL, Talpaz M, Nicaise C, Huang F, Sawyers CL (2005) Molecular analysis of dasatinib resistance mechanisms in CML patients identifies novel $B C R-A B L$ mutations predicted to retain sensitivity to imatinib: rationale for combination tyrosine kinase inhibitor therapy. Blood 106: 318a

Simonsson B (2005) Beneficial effects of cytogenetic and molecular response on long-term outcome in patients with newly diagnosed chronic myeloid leukaemia in chronic phase (CML-CP) treated with imatinib (IM): update from the IRIS Study. Blood 106: 52a

Stover EH, Chen J, Lee BH, Cools J, McDowell E, Adelsperger J, Cullen D, Coburn A, Moore SA, Okabe R, Fabbro D, Manley PW, Griffin JD, Gilliland DG (2005) The small molecule tyrosine kinase inhibitor AMN107 inhibits TEL-PDGFRbeta and FIP1L1-PDGFRalpha in vitro and in vivo. Blood 106: $3206-3213$

Von Bubnoff N, Gorantla SH, Kancha RK, Lordick F, Peschel C, Duyster J (2005) The systemic mastocytosis-specific activating c-kit mutation D816V can be inhibited by the tyrosine kinase inhibitor AMN107. Leukaemia 19: 1670-1671

Von Bubnoff N, Sanger J, Manley PW, Mestan J, Peschel C, Duyster J (2006) $\mathrm{Bcr}-\mathrm{Abl}$ resistance screening predicts a limited spectrum of point mutations to be associated with clinical resistance to the Abl kinase inhibitor AMN107. Blood 107, (Epub ahead of print)

Weisberg E, Manley PW, Breitenstein W, Bruggen J, Cowan-Jacob SW, Ray A, Huntly B, Fabbro D, Fendrich G, Hall-Meyers E, Kung AL, Mestan J, Daley GQ, Callahan L, Catley L, Cavazza C, Azam M, Neuberg D, Wright RD, Gilliland DG, Griffin JD (2005) Characterization of AMN107, a selective inhibitor of native and mutant Bcr-Abl. Cancer Cell 7: $129-141$

White DL, Saunders VA, Dang P, Engler J, Zannettino ACW, Cambareri A, Quinn S, Manley P, Hughes TP (2006) Oct-1 mediated influx is a key determinant of the intracellular uptake of imatinib but not AMN107; reduced OCT-1 activity is the cause of low in vitro sensitivity to imatinib. Blood 107 (in press) 